\title{
1 Zoonotic spillover of SARS-CoV-2: mink-adapted virus in humans
}

3 Lukasz Rabalski ${ }^{1}$ (https://orcid.org/0000-0002-5292-9263),

4 Maciej Kosinski ${ }^{1}$ (https://orcid.org/0000-0002-5475-7733),

5 Natalia Mazur-Panasiuk ${ }^{2}$ (https://orcid.org/0000-0003-1167-2622)

6 Boguslaw Szewczyk ${ }^{1}$ (https://orcid.org/0000-0003-4957-4059),

7 Krystyna Bienkowska-Szewczyk ${ }^{3}$ (https://orcid.org/0000-0003-3650-2902),

8 Ravi Kant ${ }^{4,5}$ (https://orcid.org/0000-0003-3878-9775)

9 Tarja Sironen ${ }^{4,5}$ (https://orcid.org/0000-0002-2344-2755),

10 Krysztof Pyrć ${ }^{2, *}$ (https://orcid.org/0000-0002-3867-7688),

11 Maciej Grzybek ${ }^{6, *}$ (https://orcid.org/0000-0002-8780-0304)

\section{Affiliations}

141 Laboratory of Recombinant Vaccines, Intercollegiate Faculty of Biotechnology of 15 University of Gdansk and Medical University of Gdansk, Abrahama 58, 80-307, Gdansk, 16 Poland

$17{ }^{2}$ Virogenetics Laboratory of Virology, Małopolska Centre of Biotechnology, Jagiellonian 18 University, Gronostajowa 7A, 30-387, Kraków, Poland

$19{ }^{3}$ Laboratory of Virus Molecular Biology, Intercollegiate Faculty of Biotechnology of 20 University of Gdansk and Medical University of Gdansk, Abrahama 58, 80-307, Gdansk, 21 Poland

$22{ }^{4}$ Department of Virology, University of Helsinki, Haartmaninkatu 3, Helsinki FI-00290, 23 Finland 
$24{ }^{5}$ Department of Veterinary Biosciences, University of Helsinki, Agnes Sjöbergin katu 2,

25 Helsinki FI-00790, Finland

$26{ }^{6}$ Department of Tropical Parasitology, Institute of Maritime and Tropical Medicine, Medical

27 University of Gdansk, Powstania Styczniowego 9B, 81-519, Gdynia, Poland.

29 Corresponding authors: Maciej Grzybek and Krzysztof Pyrc

30 Corresponding author emails: maciej.grzybek@gumed.edu.pl \& k.a.pyrc@uj.edu.p

\section{ABSTRACT}

32

33 The COVID-19 pandemic caused by SARS-CoV-2 started in fall 2019. A range of different 34 mammalian species, including farmed mink, have been confirmed as susceptible to infection 35 with this virus. We report here the spillover of mink-adapted SARS-CoV-2 from farmed 36 mink to humans after extensive adaptation that lasted at least 3 months. We found the 37 presence of four mutations in the $\mathrm{S}$ gene (that gave rise to variant: G75V, M177T, Y453F and $38 \mathrm{C} 1247 \mathrm{~F}$ ) and others in an isolate obtained from SARS-CoV-2 positive patient.

39

40

44 Conflict of interest: None declared

Keywords: SARS-CoV-2; Bat coronavirus; mink; transmission; spillover; zoonoses;

Expanding host range; jumping the species barrier

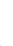




\section{Introduction}

49 Coronaviruses are known as potential zoonotic pathogens, and severe acute respiratory 50 syndrome coronavirus 2 (SARS-CoV-2) is the third highly pathogenic member of this family

51 to have emerged in the $21^{\text {st }}$ century (1). While mass vaccinations are currently underway, the

52 question of the fate of the virus remains open. The concept of herd immunity and eradication

53 of the virus is somewhat unrealistic when considering the prevalence, genetic diversity, and

54 the existing animal reservoirs. Thus far, SARS-CoV-2 infections have been reported in 55 different mammalian species worldwide, including dogs, cats, tigers, lions, ferrets, minks, 56 felines, and deer $(2,3)$. SARS-CoV-2 infections in farmed mink have recently been confirmed 57 in European countries (4). Transmission of the virus from infected mink to humans has been 58 reported in Denmark and the Netherlands (5-7). After Denmark, Poland is the second-largest 59 producer of mink pelts in Europe, with 354 active Polish mink farms harbouring 60 approximately 6.3 million mink in total (8).

\section{Material and methods}

63 This study was approved by the Independent Bioethical Committee for Scientific Research at the Medical University of Gdansk, Gdansk, Poland (Statement no. NKBBN/183/2020).

SARS-CoV-2 genome sequencing was performed at the University of Gdansk, Poland using

67 sample containing RNA isolated from positive swab (amplification of two target genes in

68 RT-PCR). ARTICv3 amplicon generation followed by Oxford Nanopore Technology

69 MinION run was performed (Quick 2020). Reads were basecalled, debarcoded and trimmed

70 to delete adapter, barcode and PCR primer sequences. ARCTIC pipeline software were used

71 to generate SARS-CoV-2 genome. Phylogenetic analysis was performed using the procedure 


\section{Results and Discussion}

74 The capacity of coronaviruses to transfer between mammalian species is not surprising. First,

75 the transfer of highly pathogenic variants from bats usually occurs via intermediate hosts.

76 Infection of dromedary camels and palm civets and later transfer to humans has been

77 described for MERS-CoV and SARS-CoV, respectively (9). A more comprehensive analysis

78 of existing strains suggests that this was not an exception. The human coronavirus OC43

79 virus (HCoV-OC43) is a betacoronavirus described for the first time in the 1960s. This

80 pathogen is associated with upper and lower respiratory tract disease in humans (10).

81 Interestingly, closely related species are found in cattle (bovine coronavirus) and dogs

82 (canine respiratory coronavirus). While the exact transfer route between different species

83 remains unknown, it is conceivable that as in SARS-CoV-2, the virus has jumped between

84 humans, and companion and farmed animals in the past (11).

85 Recently, we and others have detected SARS-CoV-2 infection in farmed minks in Northern

86 Poland $(12,13)$. The first report identified SARS-CoV-2 in samples collected from mink in

87 mid-November 2020. While the prevalence of the virus was low and, most likely, only

88 isolated cases were present, we have sequenced the isolates and have shown that the positive

89 signal did not originate from contamination. The most recent data collected and deposited by

90 the laboratory of the National Veterinary Research Institute in Poland has indicated isolation

91 of the SARS-CoV-2 variants from animals at the same farm. Phylogenetic analysis of the

92 data have indicated that the virus belongs to the B.1.1.279 lineage (Pangolin classification),

93 which is not surprising considering the prevalence of this variant in Europe. Genome analysis

94 shows that the new isolates carry the combination of mutations typical of viruses isolated

95 already in November 2020 from mink (listed in Figure 1 bottom left radial tree), but

96 additional new changes have accumulated since then. These include the Y453F mutation, 
97 which was previously reported to have emerged in minks during serial passages (e.g., in

98 Denmark and recently Lithuania), and a novel mutation not present in any global SARS-

$99 \mathrm{CoV}-2$ isolate that truncate $\mathrm{ORF} 7 \mathrm{~b}$ at position L22. With all the available data taken into

100 account, we speculate that the virus was present already in the mink population by November

1012020 , presumably after a single introduction during the late summer or autumn of that year. 
bioRxiv preprint doi: https://doi.org/10.1101/2021.03.05.433713; this version posted March 5, 2021. The copyright holder for this preprint (which was not certified by peer review) is the author/funder, who has granted bioRxiv a license to display the preprint in perpetuity. It is made available under aCC-BY-ND 4.0 International license.
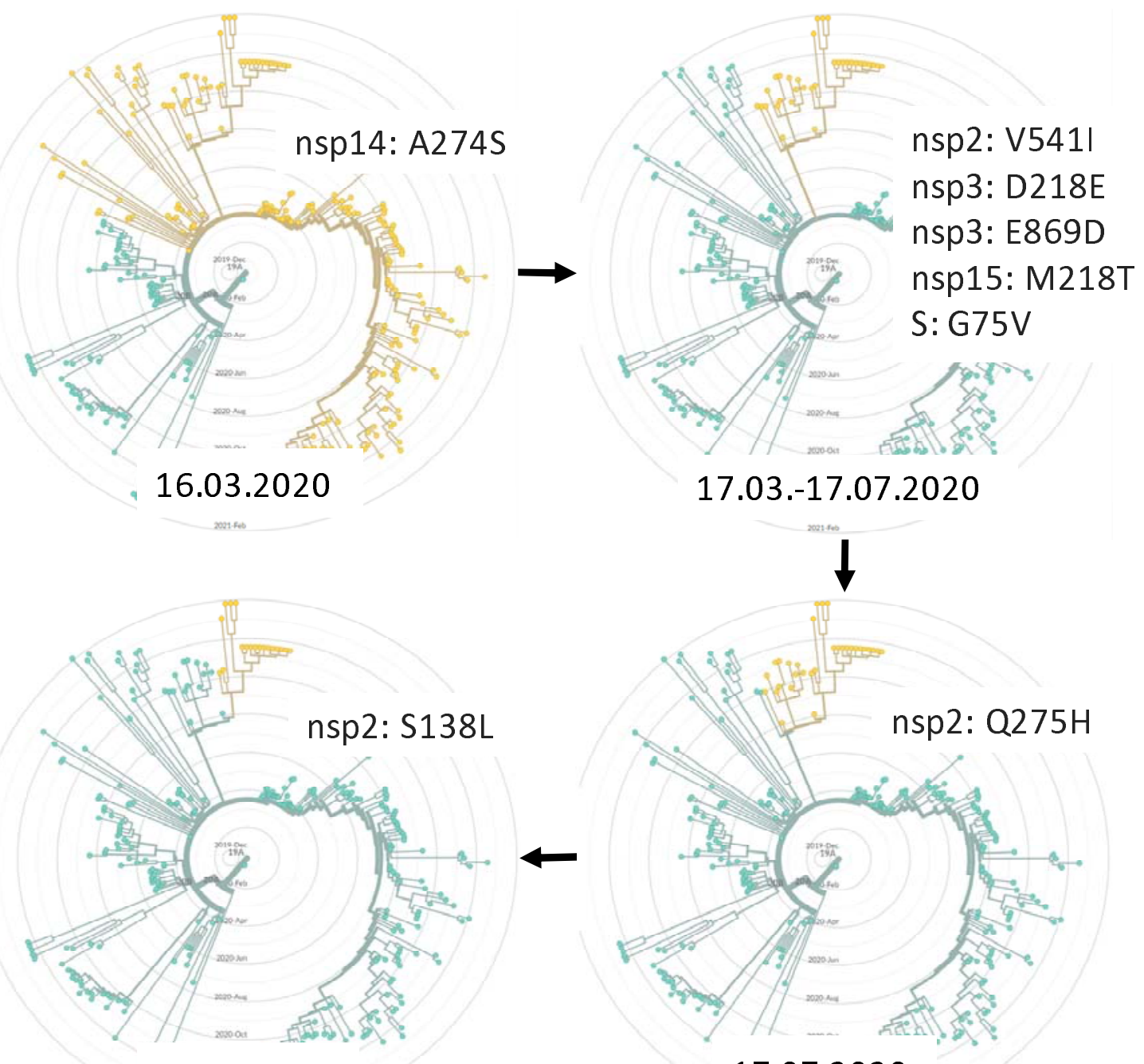

27.07.2020

17.07.2020
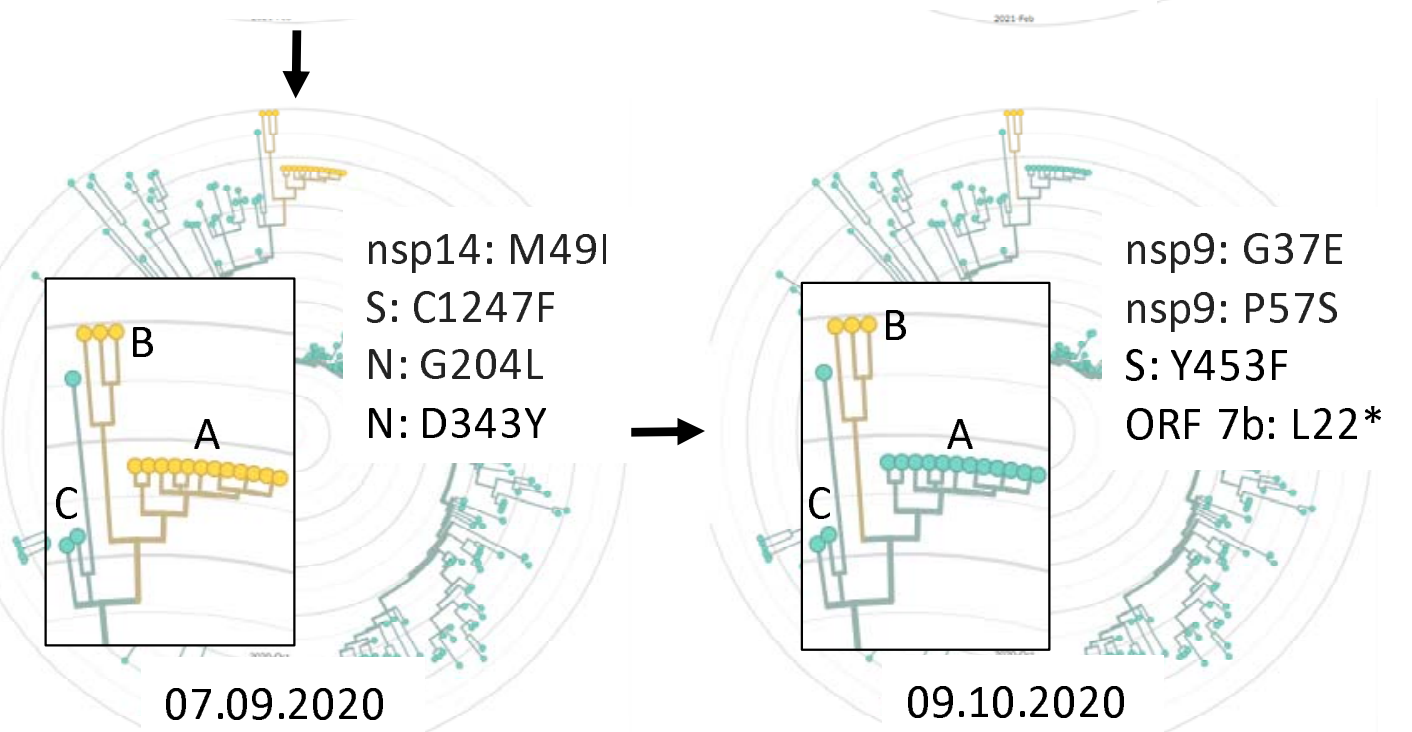
103 Figure 1. Phylogenetic analysis of SARS-CoV-2 lineage B.1.1.279 combined with inferred

104 time (bottom of each radial time tree) of fixing mutations (upper right of each radial time

105 tree) in all isolates form a group that leads to the generation of the mink variants. Yellow

106 colour represents new variants, A - November 2020 mink isolates, B - January 2021 mink

107 isolates and single human isolate, $\mathrm{C}$ - nearest neighbours human isolates that share a common

108 ancestor with A-B: Norway/4235/2020, Germany/NW-HHU-340/2020, Iceland/4563/2021.

109 In the current study, we have identified a case of infection with a mink-adapted variant in

110 humans. Following the identification of SARS-CoV-2 cases in farmed animals, the exposed

111 staff were tested for SARS-CoV-2 (nasopharyngeal swabs) using RT-qPCR. A single positive

112 case was detected in a sample collected on $1^{\text {st }}$ February 2021 and was sequenced using the

113 ARTIC Nanopore technology protocol. The resulting sequence has been deposited in

114 GISAID under the accession number EPI_ISL_1034274. The host was asymptomatic.

115 Phylogenetic analysis (Figure 1) shows that the virus clusters closely with viruses isolated

116 from mink (group B on bottom radial trees). Further, mutations characteristic of the mink-

117 adapted variant were present in the virus's genomic sequence (Table 1), confirming that it

118 was most likely contracted by the infected subject from animals.

119 Table 1. Representation of mutations in mink originated SARS-CoV-2 isolates. Group A -

120 November 2020 mink isolates, Group B - January 2021 mink isolates and single human

121 isolate, C - nearest neighbours in lineage B.1.1.279 human isolates. The yellow colour

122 represents mutations fixed in November 2020. The red colour represents mutations acquired

123 during three months of passage at the mink farm. 


\begin{tabular}{|c|c|c|c|c|c|}
\hline nt possition & aa variant & Reference & GroupA & Group B & Group C \\
\hline 1218 & nsp2: S138L & C & T & T & T \\
\hline 1630 & nsp2: Q275H & A & T & T & T \\
\hline 2426 & nsp2: V541l & G & A & A & A \\
\hline 3373 & nsp3: D218E & C & A & A & A \\
\hline 5326 & nsp3: E869D & G & T & T & T \\
\hline 12795 & nsp9: G37E & G & G & A & G \\
\hline 12854 & nsp9: P57S & C & C & T & C \\
\hline 14408 & nsp12: P323L & C & T & T & T \\
\hline 18186 & nsp14: M49I & G & T & T & G \\
\hline 18859 & nsp14: A274S & G & T & T & T \\
\hline 20273 & nsp15: M218T & T & C & C & C \\
\hline 21786 & S: G75V & G & T & T & T \\
\hline 22092 & S: M177T & T & T & C & T \\
\hline 22920 & S: Y453F & A & A & T & A \\
\hline 25302 & S: C1247F & G & T & T & G \\
\hline 27820 & ORF $7 b:$ L22 & T & T & A & T \\
\hline $28881 / 2$ & N: R203K & GG & AA & AA & AA \\
\hline 28884 & N: G204L & G & T & T & G \\
\hline 29300 & N: D343Y & G & T & T & G \\
\hline & & & & \\
\hline
\end{tabular}

125

126 The emerging variant clearly reflects adaptation of the virus to the animal host, with some

127 point mutations fixed early during transmission between animals and additional changes

128 accumulating over time (Figure 1, bottom right). While the mutations' exact role is to be

129 determined, they may provide increased fitness in the new host (14). It remains unknown

130 whether these features alter the course of disease transmissibility, or immunogenicity in

131 humans (15). However, the variant should be tracked in the general population. Our results

132 confirm the need for country-scale epizootiological monitoring and careful analysis of SARS-

133 CoV-2 positive patients.

137 1. Cucinotta D, Vanelli M. WHO Declares COVID-19 a Pandemic. Acta Biomed 
[Internet]. 2020;91(1):157-60. Available from:

http://www.ncbi.nlm.nih.gov/pubmed/32191675

140 2. Palmer M V, Martins M, Falkenberg S, Buckley A, Caserta LC, Mitchell PK, et al.

[Internet]. 2021 Jan 1;2021.01.13.426628. Available from:

http://biorxiv.org/content/early/2021/01/14/2021.01.13.426628.abstract

144 3. Abdel-Moneim AS, Abdelwhab EM. Evidence for SARS-CoV-2 Infection of Animal

Hosts. Pathogens [Internet]. 2020 Jun 30;9(7):529. Available from:

https://www.mdpi.com/2076-0817/9/7/529

147 4. World Organisation for Animal Health. World Organisation for Animal Health

[Internet]. 2021 [cited 2021 Jan 12]. Available from: https://www.oie.int/en/scientific-

expertise/specific-information-and-recommendations/questions-and-answers-on-

151 5. Hammer AS, Quaade ML, Rasmussen TB, Fonager J, Rasmussen M, Mundbjerg K, et al. SARS-CoV-2 Transmission between Mink ( Neovison vison ) and Humans,

Denmark. Emerg Infect Dis [Internet]. 2021 Feb;27(2). Available from:

http://wwwnc.cdc.gov/eid/article/27/2/20-3794_article.htm

6. Koopmans M. SARS-CoV-2 and the human-animal interface: outbreaks on mink

farms. Lancet Infect Dis [Internet]. 2020 Nov;1. Available from:

https://linkinghub.elsevier.com/retrieve/pii/S1473309920309129

158 7. Oude Munnink BB, Sikkema RS, Nieuwenhuijse DF, Molenaar RJ, Munger E,

162 8. EU Fur Association. EU Fur Association [Internet]. 2020 [cited 2020 Dec 13]. 
Available from: https://www.sustainablefur.com/

$1649 . \quad$ de Wit E, van Doremalen N, Falzarano D, Munster VJ. SARS and MERS: recent insights into emerging coronaviruses. Nat Rev Microbiol [Internet]. 2016 Aug

10. Kahn JS, McIntosh K. History and Recent Advances in Coronavirus Discovery.

Pediatr Infect Dis J [Internet]. 2005 Nov;24(11):S223-7. Available from: https://journals.lww.com/00006454-200511001-00012

170 11. Szczepanski A, Owczarek K, Bzowska M, Gula K, Drebot I, Ochman M, et al. Canine Respiratory Coronavirus, Bovine Coronavirus, and Human Coronavirus OC43:

Receptors and Attachment Factors. Viruses [Internet]. 2019 Apr 5;11(4):328.

Available from: https://www.mdpi.com/1999-4915/11/4/328

12. Rabalski L, Kosinski M, Smura T, Aaltonen K, Kant R, Sironen T, et al. Detection and molecular characterisation of SARS-CoV-2 in farmed mink (Neovison vison) in Poland. bioRxiv. 2020;

13. GISAID. GISAID [Internet]. 2021 [cited 2021 Feb 18]. Available from: https://www.epicov.org/epi3/ EPI_ISL_984305,EPI_ISL_984307

14. Zhao J, Cui W, Tian B. The Potential Intermediate Hosts for SARS-CoV-2. Front Microbiol [Internet]. 2020 Sep 30;11. Available from: https://www.frontiersin.org/article/10.3389/fmicb.2020.580137/full

15. Hayashi T, Yaegashi N, Konishi I. Effect of RBD mutation (Y453F) in spike glycoprotein of SARS-CoV-2 on neutralizing antibody affinity. bioRxiv [Internet]. 2020 Jan 1;2020.11.27.401893. Available from: 


\section{Data availability}

189 The complete genome sequences of SARS-CoV-2 isolated from the patient was deposited in

190 GISAID under the accession number: EPI_ISL_1034274

\section{Authors' contributions:}

192 The study was conceived and designed by LR and MG. Data handling: LR, MK, MG, KP.

193 Material handling and laboratory work: LR, MK, NMP. Statistical and phylogenetic analysis

194 was carried by LR. Interpretation of data: LR, KP, MK. Data visualisation: LR. Supervision:

195 MG, KP, TS. The manuscript was written by LR, MG, KP, RK, TS, BS, KBS in consultation

196 with all co-authors. MG, KP, LR, TS, RK revised the manuscript. All authors accepted the

197 final manuscript version.

198 Acknowledgements:

199 We gratefully acknowledge the Authors from the Originating laboratories responsible for

200 obtaining the specimens and the Submitting laboratories where genetic sequence data were

201 generated and shared via the GISAID Initiative, on which this research is partially based. 